\title{
Abcès mycosique cérébral humain par Cladosporium trichoïdes
}

\author{
Par Michèle DOBY-DUBOIS, Marie-Louise CHEVREL \\ Jean-Marie DOBY et Yvon ROBERT
}

Le diagnostic d'abcès cérébral causé par un champignon brun, classé dans les Dématiées, est posé plus souvent depuis quelques années. C'est en 1949, semble-t-il, que le premier cas fut fortuitement découvert, en France, après autopsie, sur coupes de cerveau (Garcin et coll.). Cependant, c'est en 1952 que Bindford et coll. aux U.S.A. (Maryland) isolaient pour la première fois le champignon en culture. Un second cas avec culture a été observé dans une autre région des U.S.A., en Pennsylvanie (King et Collette, 1952) ; le même agent pathogène, nommé par Emmons Cladosporium trichoïdes, a été isolé de ces deux mycoses cérébrales. Depuis, à notre connaissance, dix autres observations, avec culture, ont été publiées dans diverses parties du globe, prouvant que cette mycose rare est néanmoins cosmopolite : la troisième publication relate un cas du Congo belge (Lucasse et coll., 1954); le quatrième cas, diagnostiqué au Vénézuéla en 1954, est associé à un petit abcès pulmonaire (Jaffé et coll., 1955) ; le cinquième est observé en France en 1954 (Segretain et coll., 1955 ; Garcin et coll., 1957), le sixième en Belgique en 1955 (Dereymaeker et De Somer, 1955), le septième en Louisiane (troisième cas aux U.S.A.) en 1956 (Mac Gill et Brueck). Il n'est pas fait mention de l'observation japonaise (Iwata et Wada, 1957 ; Nagahata et coll., 1958) dans la bibliographie des « Cladosporioses cérébrales » de Mariat (1957), ni dans celle de Barnola et Ortega (1961), bien que l'agent ait été isolé en culture ; il ne s'agit plus ici de l'espèce neurotrope identifiée et décrite par Emmons (1952), mais d'un champignon responsable de chromoblastomycose de la peau, Hormodendrum pedrosoï (1) ; c'est la première observation d'abcès métastatiques du cerveau, trouvés à l'autopsie, faisant suite à une chromomycose de la face (2). La neuvième publication relate un cas de cladosporiose cérébrale prouvée par la culture d'une espèce d'Hormodendrum (1) observé en Afrique du Sud (Watson

(1) Hormodendrum, dénomination générique des auteurs.

(2) Cependant la culture de Lucasse et coll. fut étudiée par la suite par Borelli qui la classa Fonsecaea pedrosoï (Duque, 1961). 
et Lines, 1957). La même année, aux U.S.A., la dixième diagnose avec culture est faite, quatrième dans ce pays (Riley et Mann, 1960).

Cladosporium trichoïdes est à nouveau isolé en France, à Lyon en 1961, d'abcès cérébral (Coudert, 1961).

De deux autres cas survenus en Amérique du Sud en 1959 (Duque, 1961), l'un, opéré avec succès, a permis d'isoler la dixième souche identifiée Cladosporium trichoïdes Emmons 1952, portant à 12 le nombre des cultures réalisées.

Plusieurs autres cas de mycose diagnostiqués sur coupe de cerveau, après autopsie ou extraction chirurgicale, semblent présenter à l'examen histopathologique les mêmes caractéristiques que les cas de cladosporiose précédemment cités et ont été considérés comme tels par leurs auteurs.

L'ensemble de ces publications est résumé dans le tableau ci-joint.

A Rennes, en 1961, l'ensemencement de pus mycosique, prélevé dans un abcès cérébral (3), nous a permis d'isoler en culture un champignon noirverdâtre velouté, ressemblant aux descriptions d'autres souches de l'espèce C. trichoïdes Emmons. Nous entretenons d'ailleurs en mycothèque deux souches isolées à l'Institut Pasteur de Paris et une à Lyon, avec lesquelles nous avons pu comparer la nôtre.

\section{Origine du matériel d'étude}

Le matériel ayant permis l'isolement de la souche était constitué par un prélèvement de tissu cervical renfermant en sa partie médiane un abcès. L'intervention chirurgicale (4) avait été effectuée sur un homme adulte, originaire de la Sarthe, hospitalisé d'urgence pour des manifestations paralytiques au niveau du bras droit et de la face, avec obnubilation et aphasie.

A l'intervention, le cerveau était extrêmement mou et œdémateux. Au niveau de la partie postérieure de F1-F2, à $3 \mathrm{~cm}$. de profondeur, une poche à paroi épaisse, du volume d'une grosse noix, renfermait un liquide purulent, du sang et du tissu cérébral nécrotique. C'était la seule lésion organisée dont l'exérèse était possible. Une vaste zone nécrosée et ramollie, occupant toute la partie basse et profonde postérieure du lobe frontal, dut être aspirée au suceur. Le malade décéda trois jours plus tard.

\section{Examen histo-pathologique}

L'examen a montré au centre du prélèvement une importante cavité dans laquelle existait un magma nécrotique bordé par des éléments histiocytaires avec nombreuses cellules multinucléées, auxquels se mêlaient des éléments blancs, lymphocytes et polynucléaires. Au niveau du magma nécrotique et de la paroi de l'abcès, étaient visibles des filaments mycéliens. Les colorations bactériologiques sont restées négatives.

(3) Conf. Chevrel et coll., 1962.

(4) Service de Neuro-Chirurgie (Prof. Pecker, Doct. Javalet) du Centre Hospitalier Régional de Rennes. 
La diagnose mycologique de cladosporiose cérébrale repose à la fois sur l'examen microscopique de frottis de pus et de coupes d'abcès, l'examen macroscopique et microscopique des cultures en tubes ou sur lames et l'inoculation expérimentale. Nous décrirons donc successivement les caractères du champignon isolé à Rennes dans la mycose humaine ou animale et en culture.

\section{Le champignon à l'état parasitaire}

\section{Coupes d'abcès fermé.}

\section{MYCOSE HUMAINE}

La recherche bactériologique par culture de pièce opératoire n'avait pas permis d'isoler de germe pathogène. Cependant, sur les préparations de coupes à la paraffine colorées par la méthode classique à l'hémalun-éosine, apparaissaient nombreux, fragmentés, des filaments irréguliers prenant plus ou moins bien le colorant. En effet, le mycélium septé possède son propre pigment, brunâtre au microscope, que la coloration artificielle ne change pas beaucoup ; mais sa seule réfringence et sa coloration naturelle permettent de le distinguer aisément sur une coupe peu colorée. Par contre, les éléments fongiques se colorent parfaitement au réactif de Schiff par la technique de Hotchkiss-Mac Manus, modifiée par Kligman et Mescon (1950).

Les hyphes, parfois ramifiés, ont une paroi lisse et de coloration brun clair ; ils sont abondants au voisinage des hématies et dans la zone moyenne de la paroi de l'abcès, au voisinage de la masse centrale purulente ou nécrotique. $\mathrm{La}$ plupart sont segmentés en éléments droits de 6 à $15 \mu$ de longueur, à section parfois légèrement ovalaire de $1 \mu 5$ à $4 \mu$ de diamètre, le plus souvent 2 à $3 \mu$. $\mathrm{Ce}$ sont quelquefois des « articles courts, plus ou moins renflés, ovoïdes ou en tonnelet \$ (Segretain et coll., 1955), disposés en chapelet sinueux. Les cellules renflées en "vésicules " sont assez nombreuses, parfois isolées en petits groupes, parfois bourgeonnantes disposées sur un trajet filamenteux; elles mesurent 5 à $8 \mu$ de diamètre et ont une paroi lisse et épaisse. Nous n'en avons trouvé aucune à surface nettement rugueuse noire, appelées par Silva (1957) " sclerotic cells ", mais nous avons vu quelques rares éléments noirâtres de forme mal définie, réfringents, et parfois de petites granulations noirâtres à l'intérieur des polynucléaires, éléments pigmentés déjà signalés par Wybel (1952).

\section{Frottis de pus.}

Le pus, gluant et sanguinolent, a été prélevé à l'intérieur d'un abcès fermé quelques jours après l'extraction chirurgicale. Cette substance jaunâtre sale, 
épaisse, grumeleuse, présente dans sa masse des magmas gris-verdâtre de structure filamenteuse, mycélienne, visible par écrasement entre lame et lamelle. Dans ces amas mycéliens, les hyphes à segments droits sont nombreux, de 10 à $30 \mu$ de long, de $2 \mu$ à $3 \mu$ de diamètre ; les « vésicules 》 arrondies, souvent régulières ici, sont uniques ou en séries de trois ou quatre sur les trajets mycéliens et peuvent atteindre $10 \mu$ de diamètre ; elles sont parfois à l'origine d'une ou plusieurs ramifications et contiennent un cytoplasme granuleux très réfringent.

Les caractères du champignon in situ permettent de rapprocher ce cas des autres cas de cladosporiose cérébrale à culture positive. Cependant on peut remarquer, comme Vanbreuseghem en 1954 (Lucasse et coll.), un diamètre plus grand du mycélium ( 2 à $3 \mu$ ) que dans le premier cas américain ou le premier cas français $(1 \mu 5)(5)$; nous n'avons pas trouvé de vésicules septées décrites par ce même auteur, entre autres, mais seulement une chlamydospore à paroi épaisse un peu rugueuse d'où partait une succession régulière, mais sinueuse, d'articles en tonnelet.

La coupe histologique de pièce opératoire, dans les publications précédentes, révèle une * prolifération granulomateuse diffuse avec cellules géantes contenant des fragments d'hyphes, fibroblastes et lymphocytes » (Barnola et Ortéga, 1961). Une technique de coloration à l'hémalun nous a permis également de mettre en évidence des cellules à noyaux multiples contenant des éléments mycéliens. « La paroi de l'abcès est épaisse de $5 \mathrm{~mm}$. »..., \& le centre est mou, grisâtre ou noirâtre, et contient de nombreuses structures filamenteuses » (Emmons, 1952).

\section{Le champignon à l'état saprophytique}

\section{Caractères culturaux.}

Dès leur apparition, les colonies, noir-verdâtre d'emblée en culture pure, rappelaient, par leur aspect macroscopique et microscopique, les champignons des chromoblastomycoses, dont nous entretenons quelques souches en mycothèque.

Des fragments de pus, lavés dans du liquide de Raulin, ont été ensemencés en culture primaire sur une série de tubes de différents milieux gélosés; la croissance fut rapide et tous ont produit des colonies, noires et vert-noirâtre,

(5) Néanmoins, quelques remarques s'imposent :

$1^{\circ}$ Emmons a mesuré le diamètre des hyphes sur matériel fixé et coloré, donc plus ou moins rétracté et déshydraté.

$2^{\circ}$ Ségrétain et coll. (1955) ont décrit dans le pus frais des hyphes ayant un diamètre moyen de $1,50 \mu$, mais atteignant parfois 3 ou $3,30 \mu$.

$3^{\circ}$ Ces mêmes auteurs décrivent dans les cultures des filaments dont le diamètre varie entre 2 et $3 \mu$. 
dont le mycélium aérien, épais et dru, quand il existait, était vert olive. En milieu liquide, la pullulation de colonies transparentes sphériques, verdâtres, fut presque immédiate.

En milieu de Sabouraud modifié (glucosé à $2 \%$ ), les colonies, vert sombre d'abord en surface, ont un épais duvet dressé au centre surélevé et incliné vers la périphérie, s'étalant en pente douce régulière; leur aspect est velouté brillant ; la face inférieure sous la gélose est noir intense. Le duvet se feutre ensuite en un réseau de filaments aériens sporuleux de dominante brune, à reflets parfois violacés sur les vieilles cultures.

Le milieu de Mathur (6), qui contient des sels minéraux et beaucoup moins de glucose, fait paraître le pigment plus brillant au revers, noir d'encre.

Les milieux contenant des produits biologiques ou plus complexes (P.C.B., bouillon glycériné, V.F. gélosés...) font croître des colonies multiples, de tendance moniliforme plus ou moins marquée: elles sont soit assez étroites, noires, centrées d'un duvet court, corémié, dressé, soit même tout à fait glabres, muriformes, très petites et verruqueuses, se multipliant dans la profondeur du milieu en innombrables granulations noires compactes et même dures (V.F.). En outre, la coloration du milieu P.C.B. vire du vert au noir.

Les subcultures obtenues, sur milieu glucosé et au moût de bière, après inoculation de matériel pathologique à la souris, ont les mêmes caractères macroscopiques que les cultures primaires du pus humain.

Les cultures secondaires sur les mêmes milieux se sont, elles, révélées moins vertes, le pigment prenant davantage ou plus vite la nuance brune violacée des trois souches de référence (7) ou de nos cultures primaires vieillies. Néanmoins, on peut remarquer de légères différences de coloration ou de conformation du mycélium aérien entre les cultures simultanées de ces quatre souches, même lorsqu'elles sont ensemencées ensemble sur une même boîte de Pétri ; il est probable que le degré d'ancienneté de la souche n'est pas étranger à ce fait, car les deux souches de Paris, les plus anciennes, sont plus claires, celle de Lyon plus brune et plus foncée. Dans les premiers repiquages mêmes de la souche isolée à Rennes, la pigmentation a perdu un peu de son intensité.

Sur milieux naturels, en particulier sur morceau de pomme de terre, toutes les souches sont rapidement noir-brun verruqueuses.

(6) Composition du milieu de Mathur (1950), communiquée par l'Institut de Recherches Agronomiques de Versailles, pour 1 litre :

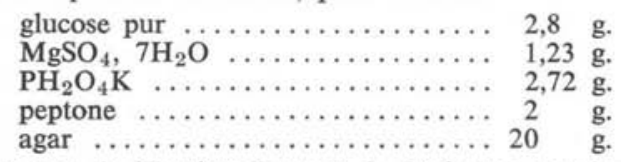

(7) Nous avons reçu de l'Institut Pasteur de Paris une souche de Cladosporium trichoïdes Emmons 1952, isolée de la mycose cérébrale publiée en 1955 ( $\left.\mathrm{n}^{\circ} 509\right)$. Une autre, originaire de l'Institut Pasteur également, mais entretenue en mycothèque au Muséum, nous a été confiée par le Professeur R. Heim ( $\left.{ }^{\circ} 1.532\right)$. La troisième, isolée récemment à Lyon, nous fut envoyée par le Professeur Coudert. Nous les remercions vivement. 
Sur le milieu liquide glucosé, il se forme d'abord une membrane, à surface plus ou moins duveteuse ou glabre, dont le pourtour adhère bientôt aux parois de verre, au-dessus de la surface du liquide ; puis le centre se creuse en entonnoir et s'enfonce en s'accroissant au fur et à mesure que le niveau du liquide baisse dans le tube. Le champignon est entièrement noir, à surface gris souris.

La croissance sur milieu à la caséine hydrolysée (Georg et Camp, 1957) est identique pour les quatre souches et beaucoup plus développée et duveteuse lorsque ce milieu contient en outre inositol et thiamine; la coloration de surface est alors typiquement « gris souris ».

\section{Caractères microscopiques.}

Les caractères microscopiques et macroscopiques s'expliquent les uns par les autres, et nous retrouvons les mêmes différences ou les mêmes analogies entre les différentes souches.

Il faut tout de suite faire la distinction entre les cultures sur milieu de Sabouraud (modifié ou non) et sur milieux peu ou pas sucrés, tels que le milieu de Mathur que nous avons préféré au milieu de Czapek pour les cultures sur lames, en raison de sa formule simple et de sa faible concentration en ose $(2,8 \%)$.

En effet, le milieu glucosé à $2 \%$ en culture sur lame produit des colonies plus épaisses dont le duvet aérien, touffu et feutré, porte d'abondantes conidies. La partie centrale en est pour cette raison peu lisible. Ces conidies naissent l'une de l'autre en longues chaînes simples, parfois ramifiées, sur des conidiophores perpendiculaires au mycélium végétatif. Ce type de fructification est appelé couramment \& forme Hormodendrum longue », celui que Vanbreuseghem appelle * forme Hormodendrum simplifiée » (Lucasse et coll., 1954), seule décrite habituellement pour l'espèce Cladosporium trichoïdes Emmons 1952. La souche rennaise présente les mêmes particularités que la souche de Paris (1955) :

1. Le diamètre des hyphes ( 2 à $3 \mu)$ et des conidies ( 3 à $4 \mu)$ est légèrement supérieur aux mesures indiquées par Emmons (1 à $2 \mu$ et 2 à $3 \mu$ ).

2. Elle produit également des hyphes enroulés en anneaux simples ou superposés par deux ou trois en une sorte de spirale tassée; ces formations que les auteurs (Segretain et coll.) rapprochent du « primordium $\gg$ de pycnides glomérulaires, existant dans les formes parfaites des Ascomycètes de l'ordre des Sphériales (8), se rencontrent à la périphérie des colonies de 4 à 5 semaines cultivées sur milieu de Mathur ou sur milieu P.C. Parfois se greffent, per-

(8) Les Cladosporium auraient une forme parfaite dans la famille des Sphériacées, par exemple Mycosphaerella tassiana étant celle de Cladosporium herbarum, selon Karsten (1872) entre autres (in de Vries, 1952). 
pendiculairement à la paroi du filament enroulé en spirale, un ou plusieurs sporophores simples à courte chaîne de spores.

En outre, sur ce même milieu de Mathur, une culture sur lame, à l'âge de six semaines, a produit des sortes de grosses cellules vésiculaires d'abord claires, à paroi épaisse, terminée par un éperon court ou un bourgeon filamenteux ; la surface lisse semble marquée parfois d'une légère arête longitudinale; d'autres sont septées asymétriquement et leur membrane est plus ou moins noirâtre. Elles sont situées sur une partie terminale de filament, souvent entre deux autres jeunes filaments peu septés et presque parallèles. Dans les zones de culture plus épaisses, on peut trouver des « chlamydospores » très sombres. En effet, sur le trajet des longues chaînes conidiennes s'intercalent parfois de grosses cellules à paroi épaisse, simples ou segmentées en diagonale, brun-noir, qui correspondent aux « chlamydospores 》 de Vanbreuseghem ou aux « sclerotic cells » dont Silva (1957) a obtenu la formation sur milieux organiques divers. Parmi les cultures isolées de cladosporioses du cerveau, celle du Congo belge (Lucasse et coll., 1954) est la seule pour laquelle on ait signalé qu'un « septum transverse pouvait diviser ces cellules ».

La bile du milieu P.C.B. favorise, semble-t-il, la production de ces \& chlamydospores » et d'une autre forme que Tréjos (1954) a trouvée dans les cultures de Cladosporium carrionii et qu'il nomme \& Chlamydospore-like elements »; les dimensions de ces dernières sont sensiblement les mêmes que celles des chlamydospores décrites ci-dessus : 8 à $10 \mu$ de diamètre; leur forme est régulière, ovalaire ; à l'intérieur de la membrane, épaisse, est régulièrement disposée une suite de cellules égales, épousant la forme des parois internes. Elles sont situées sur des trajets filamenteux entre deux files d'arthrospores ou de segments courts.

Leur signification n'est pas encore connue, leur nombre dépend de la composition du milieu de culture en produits organiques, ainsi que pour les « sclerotic cells » de Silva (1957).

Outre les chlamydospores et formes assimilées et les fructifications de \& type Hormodendrum simplifié » (type Cladosporium pour certains auteurs), nous avons trouvé, dans quelques préparations de culture en milieu P.C.B., des sporophores de « type Hormodendrum court »; Vanbreuseghem (Lucasse et coll., 1954) les classe : « $2^{\circ}$ type de fructification \$; la \& forme 4 » de ce même auteur est très rare, en culture de deux ou trois mois sur P.C.B. ; nous n'avons pas rencontré la forme 3 * acrotheca » (9); la souche rennaise là

(9) $\mathrm{La} \approx 4^{\circ}$ forme de fructification » de Vanbreuseghem, non dénommée, semble un compromis entre la forme de reproduction de type « acrotheca $»$ et celle de type « Hormodendrum » (dit « court »), mais comporte des cellules de forme particulière à gros bout distal.

La forme «acrotheca », classiquement décrite et présente dans la culture de Phialophora pedrosoï, n'est pas constamment rencontrée dans la culture de Cladosporium trichoïdes. Par leur rapide accroissement, les conidies nombreuses dessinent avec la cellule terminale qui les porte une sorte de plumet dont la tige, une fois dépouillée, aurait la forme d'une sorte de « carotte de sapin \$ (Lucasse et coll., 1954). 
encore correspond tout à fait aux descriptions de la souche de Garcin (Segretain et coll., 1955). Nous croyons pouvoir la classer, comme cette dernière, dans le cadre de l'espèce Cladosporium trichoïdes Emmons 1952. Il reste entendu cependant que l'espèce Cladosporium carrionii Tréjos, 1954, est morphologiquement très voisine, les principales différences spécifiques résidant dans le calcul statistique des longueurs moyennes des spores et le neuro- ou le dermo-tropisme.

Devant la difficulté d'apprécier en certitude la valeur spécifique de beaucoup des caractères décrits plus haut, d'autres auteurs se sont limités à l'appellation plus vague de «Hormodendrum species 》 (10), étant donné la multiplicité des noms d'espèces (11) connus dans le genre Cladosporium, dont la signification même a souvent varié avant l'important travail de thèse de De Vries (1952).

\section{Inoculations expérimentales :}

Deux souris blanches ont été inoculées par voie intrapéritonéale avec chacune un quart de centimètre cube de pus obtenu à partir de la lésion humaine. L'examen de la première souris, effectué 20 jours après l'inoculation, n'a permis de réisoler le champignon qu'à partir d'un petit nodule au point d'inoculation. Tous les organes, encéphale compris, étaient restés sains. Le germe n'a pu être réisolé de la seconde souris, examinée 30 jours après l'inoculation, même pas au niveau du point de celle-ci (12).

Une souris à laquelle fut injecté le pus par voie intracranienne mourut le $7^{e}$ jour après inoculation, avec fonte purulente du dessus du crâne dans la région inoculée et envahissement de tout un hémisphère cérébral par une masse gris-verdâtre sans contour précis ; bien que moins atteint macroscopiquement, l'autre hémisphère était envahi de mycélium brun caractéristique prenant intensément la coloration par la technique de Hotchkiss-Mac Manus. Le mycélium pathogène avait envahi aussi l'os du rocher, le cervelet, et s'était propagé sur la nuque, produisant une lésion granulomateuse sous-cutanée apparaissant

(10) « Hormodendrum » est, selon Emmons, le synonyme non valable de Cladosporium, et l'ancien nom de genre des agents des chromoblastomycoses (in Mac Gill et Brueck, 1956, et in Watson et Lines, 1957).

(11) Plus de 250 décrites, incomplètement, par Saccardo (in Bindford et coll., 1952).

(12) Dans un travail en cours sur le pouvoir pathogène comparé d'une dizaine de souches de Cladosporium ou de germes voisins, en fonction des voies d'inoculation, nous avons cependant observé, chez des souris inoculées depuis respectivement 15, 21 et 35 jours avec cette souche, non plus sous forme de pus, mais de suspension de culture en milieu liquide, la persistance et la multiplication du germe, qui détermine la formation de multiples petits granulomes répartis dans toute la cavité abdominale. De nombreux auteurs ont pourtant noté la disparition plus ou moins rapide du germe après inoculation par cette voie. 


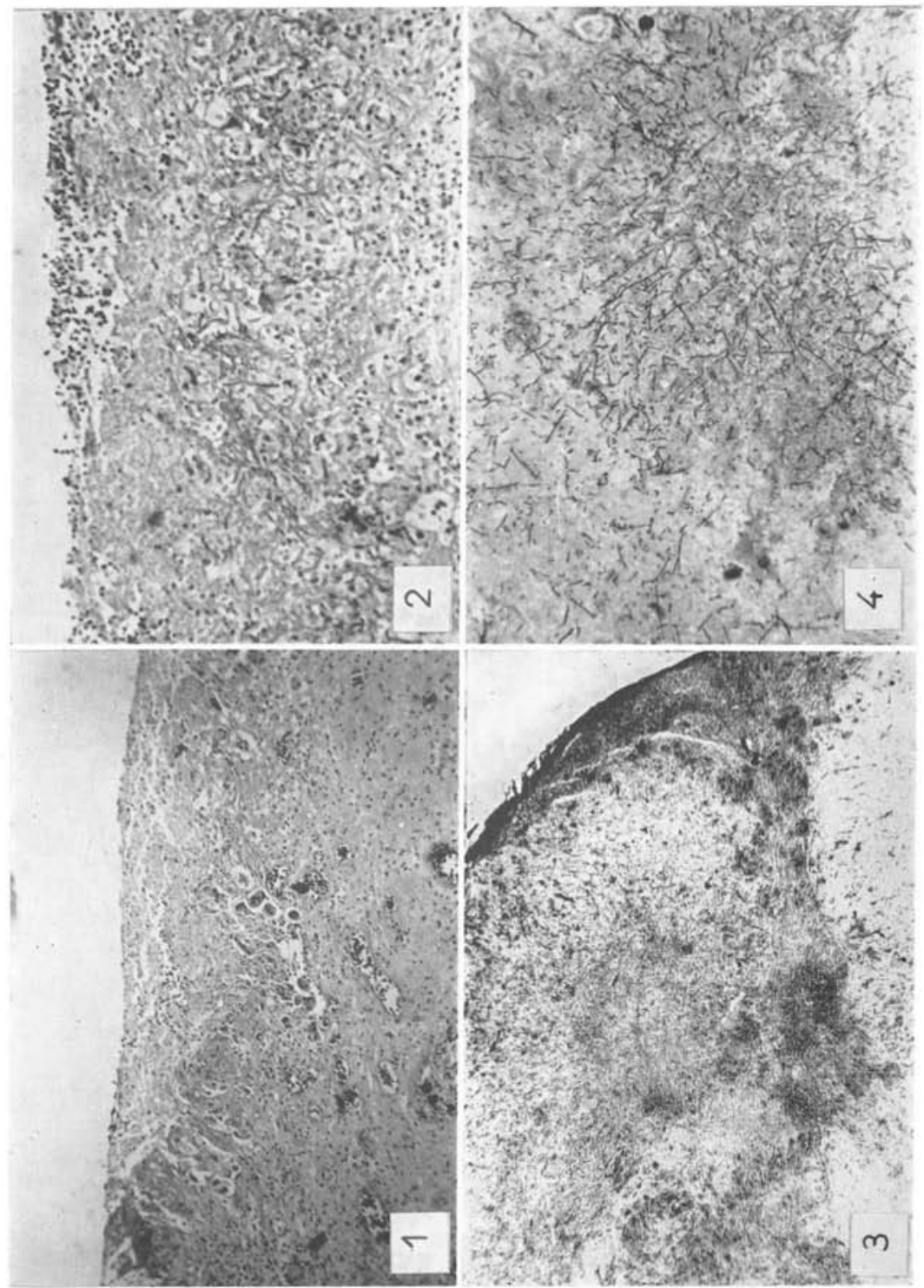

Planche I

FIG. 1 et 2. - Abcès cérébral humain. Coupes colorées au Gram Weigert. - Fig. 3 et 4. - Mycose cérébrale expérimentale. Coupe de cerveau de souris 7 jours après inoculation intracranienne. Coloration de Hotchkiss Mac Manus. 


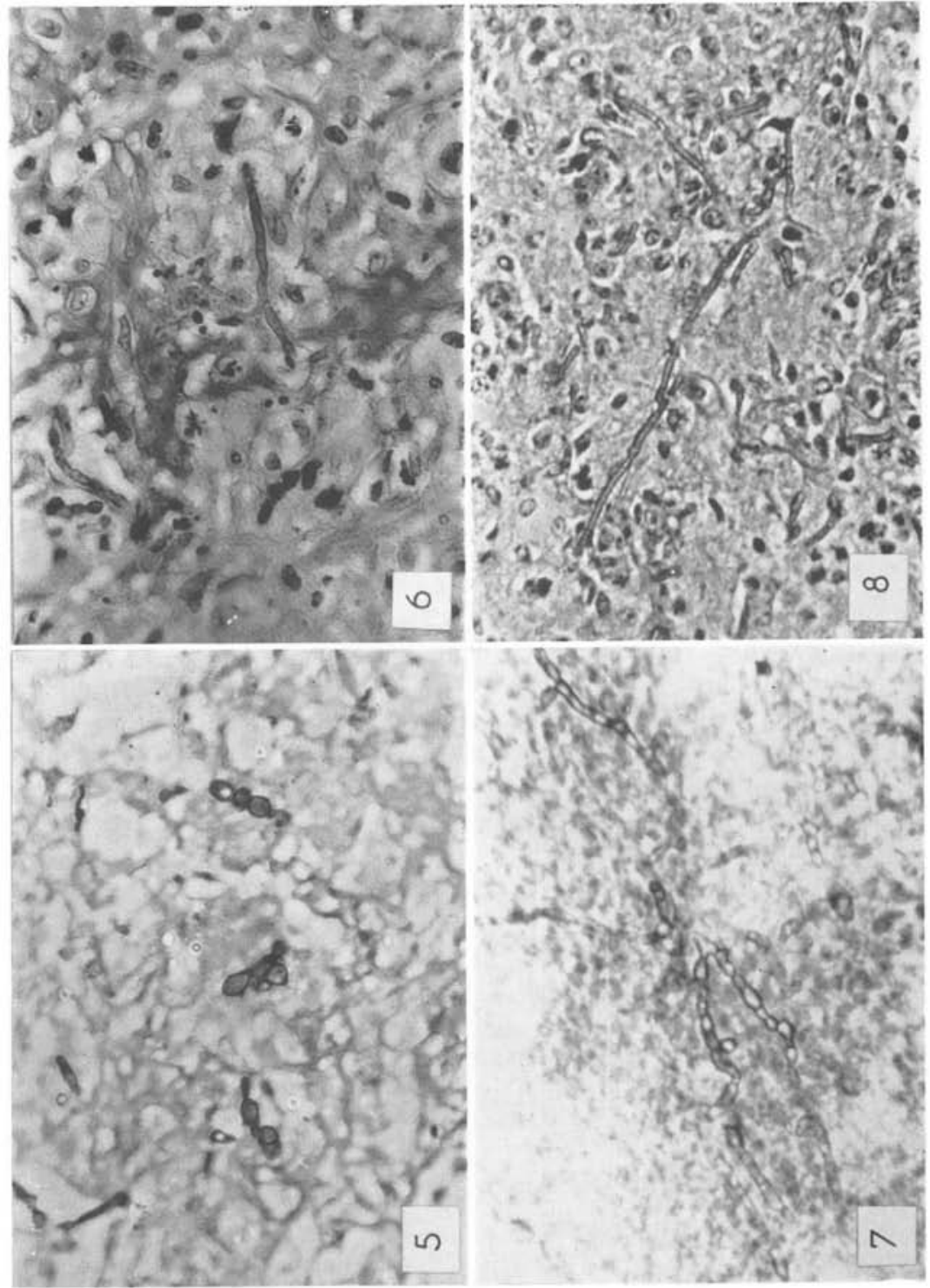

Planche II

FIG. 5 et 6. - Mycose cérébrale humaine. Détail. Coloration de Hotchkiss Mac Manus. - FIG. 7 et 8 . - Mycose cérébrale de la souris. Substance purulente et coupe de cerveau. 


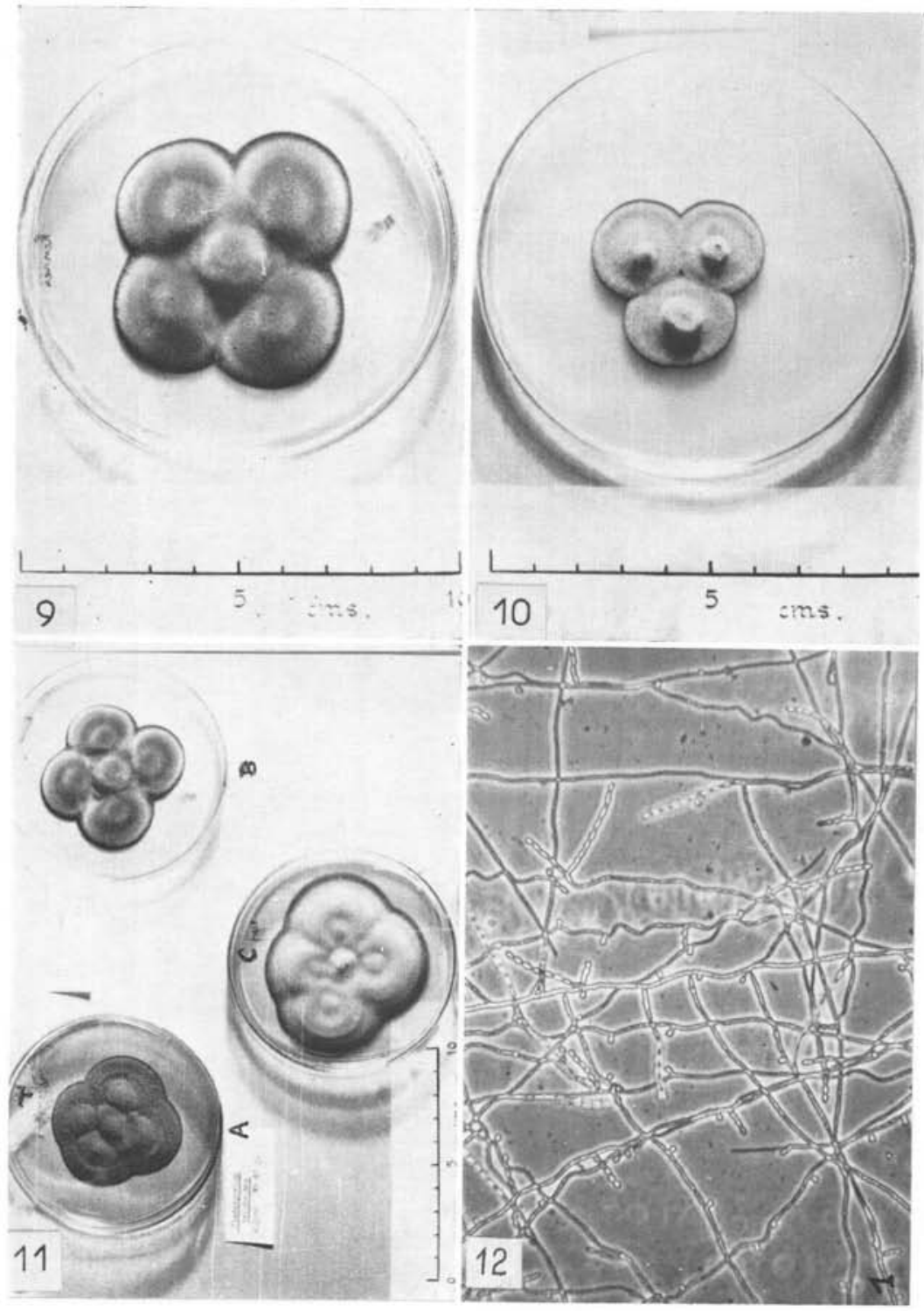

Planche III

Fig. 9. - Culture de 40 jours sur gélose au moût de bière. - Fig. 10. - Culture de 15 jours sur glucosé à $2 \%$. - Fig. 11. - Trois souches de Cladosporium sur gélose au moût de bière. 40 jours : A) Souche de $\mathrm{Cl}$. trichoïdes Lyon. - B) Souche de Cladosporium de Rennes. - C) Souche de Cl. trichoïdes de Paris (Muséum $\mathrm{n}^{\circ}$ 1532). - FIG. 12. - Aspect microscopique de la souche de Rennes. Culture sur lame d'un mois sur milieu de Mathur. 


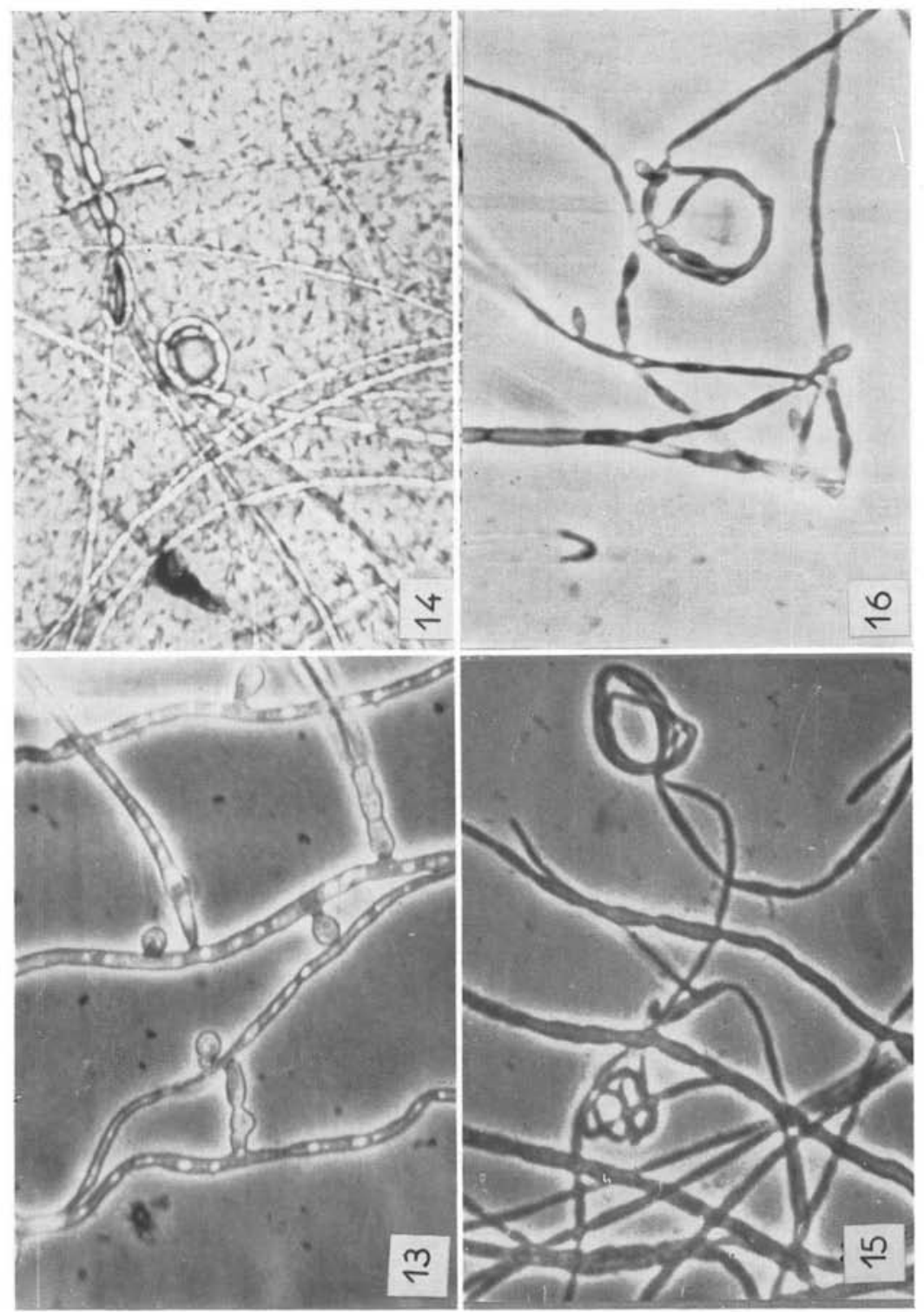

PLANCHE IV

Fig. 13. - Aspect microscopique de culture sur lame sur milieu de Mathur. Formation de chaînes conidiennes et d'anastomoses. Hyphes contenant des globules réfringents. Examen dans le bleu de toluidine-désogène au contraste de phase. FIG. 14. - Spirales à un seul anneau (diamètre de $15 \mu$ environ) et départ de chaîne conidienne. Examen dans le lactophénol. - Fig. 15 et 16 . - Spirales à plusieurs tours. Même culture. 
en noir sous la peau ; nous avons ensemencé sur gélose nutritive cette substance noir-verdâtre, friable et granuleuse, non purulente; le même champignon obtenu à partir du pus humain ou de la substance cérébrale de la souris s'est développé.

A partir de cette souris furent ensemencés sans succès, sur gélose nutritive : le liquide péritonéal, le sang prélevé dans le cœur, des fragments de poumons, de foie, de rate. Il semble donc qu'il n'y ait pas eu dissémination du parasite dans l'organisme, pas de diffusion par voie sanguine.

Pourtant, dans la lésion humaine, le transfert du parasite par voie sanguine est vraisemblable, mais la porte d'entrée reste incertaine et présente de nombreuses possibilités. Pour les cas de cladosporiose cérébrale publiés, traumatisme superficiel et inhalation sont les voies d'entrée le plus souvent suggérées, bien qu'il n'ait pas souvent été possible de les déceler avec certitude.

En règle générale, aucun autre organe que le cerveau lui-même ne semblait lésé par le champignon ; néanmoins, le deuxième cas avec culture de la littérature avait subi un drainage de l'oreille gauche avant d'être opéré d'un abcès cérébral du lobe frontal gauche; le septième cas était celui d'une femme ayant souffert plusieurs fois de traumatismes du cuir chevelu, d'abcès sous-unguéal et d'ulcère de la jambe entre autres. Le cas de Manson (1958) faisait suite également à des blessures superficielles de la tête. Par ailleurs, un cas mortel faisait suite à une chromomycose de la face (Iwata et Wada, 1957), deux autres cas (Jaffe et coll., et Horn et coll.) présentaient simultanément un abcès mycosique pulmonaire décelé à l'autopsie.

Dans la littérature médicale concernant Cladosporium trichoïdes, les inoculations expérimentales n'ont permis d'obtenir des lésions cérébrales que lorsqu'elles ont été effectuées soit directement par voie intracranienne, soit par voie intraveineuse (veine de l'oreille du lapin, veine caudale de la souris), avec du pus de lésion ou une suspension de culture. Il semble que, jusqu'à maintenant du moins, ces observations soient valables pour notre souche.

Il convient de noter que la lésion cérébrale expérimentale n'est pas enkystée et que la prolifération mycélienne s'étend à tout le cerveau et même au cervelet, accompagnée seulement d'une multiplication des noyaux cellulaires comme dans la lésion humaine.

En attendant les résultats d'une étude plus complète du pouvoir pathogène, nous croyons donc pouvoir dénommer cette souche Cladosporium trichoïdes Emmons, 1952.

\section{Bibliographie}

Barnola (J. D.) et Ortega (A. A.), 1961. - Cladosporiosis profunda. Mycopathologia et Mycologia applicata, 15, 422.

Bindford (C. H.), Thompson (R. K.) et Gorham (M. E.), 1952. - Mycotic brain abscess due to Cladosporium trichoïdes, a new species. Report of a case, with mycologic report by Emmons (C. W.). Amer. Jl Clin. Path., 22, 535. 
Bobra (S. T.), 1958. - Mycotic abscess of the brain probably due to Cladosporium trichoïdes. Report of the fifth case. Canad. Med. Ass. J., 79, 657.

Bonne (C.), Bros (G.) et VermaArt (W. J. C.), 1948. - Localised lesions of the meninges and the brain caused by a brown fungus with septate hyphae of unknown nature. Med. Maanblad. Batavia, 23, 465 (in Mariat, 1957).

Borelli (D.), 1956. - Cenni di Micopatologia Venezuelana. Giornale Italiano di Derm. e Sifil., 117, 507.

Chevrel (M.-L.), Javalet (A.), Doby (J.-M.), Doby-Dubois (M.) et Louvet (M.), 1962. - A propos d'une observation de cladosporiose cérébrale. Ann. d'Anatomie Pathologique (sous presse).

COUdert (J.), 1961. - Communication verbale.

Coudert (J.), Allegre (G.), Tomasi (M.) et Battesti (M.-R.). 1962. Un cas lyonnais de mycose cérébrale due à Cladosporium trichoïdes. Etude mycologique. Bull. de la Société Fr. de Mycologie Méd., n 4, p. 4.

Dereymaeker (A.) et DE Somer (P.), 1955. - Arachnoïdite fibropurulente cérébellocervicale due à une moisissure. Acta neurol. psychiatr. belgica, 55, 629.

DE VRIES (G.-A.), 1952. - Contribution to the knowledge of the Genus \& Cladosporium », Link ex Fries. Thèse Baarn, éditée par Centraal Bureau voor Schimmel cultures, Baarn.

Duque (O.), 1961. - Meningo-encephalitis and brain abscess caused by Cladosporium and Fonsecaea. Review of the literature, report of two cases and experimental studies. Amer. J. Clin. Path., 36, 505.

Fukushiro (R.), Kagawa (S.), Nishiyama (S.) et Takahashi (H.), 1957. - Un cas de chromoblastomycose cutanée avec métastase cérébrale mortelle. Presse Médicale, 65, 2142.

Garcin (R.), Martin (R.), Bertrand (I.), Grüner (J.) et Tourneur (R.), 1949. Mycose méningo-épendymaire. Etude anatomo-clinique. La Presse Médicale, 57, 1201, et Revue neurologique, 81, 968.

Garcin (R.), Martin (R.), Manigand (G.), Godlewski (S.) et Sureau (B.), 14 juin 1957. - Mycose cérébrale à Ciadosporium trichoïdes. Semaine des Hôpitaux, 33 A, $\mathrm{n}^{\circ} 36$ - La Sem. des Hôp. de Paris, $\mathrm{n}^{\circ}$ 57, 2282.

Georg (L. K.) et CAMP (L. B.), 1957. - Routine nutritional tests for the identification of dermatophytes. $J l$ of Bact., 74, 113.

Horn (I. H.), Wilansky (D. L.), Harland (W. A.), BlanK (F.), 1960. - Neurogenic hypernatremia with mycotic brain granulomas due to Cladosporium trichoïdes. Canad. Med. Ass. J., 83, 1314.

IWATA (K.) et WADA (T.), 1957. - Mycological studies on the strains isolated from a case of chromoblastomycosis with a metastasis in central nervous system. The Japan. Jl of Microbiology, 1, 355.

JAfFé (R.), Barnola (J.), Rivas (M.) et Martinez-Niochet (A.), février 1955. $I I I^{e}$ Journées d'Anatomie pathologique, Maracaïbo (in Barnola et Ortéga, 1961 - in Borelli, 1956).

KING (A. B.) et Collette (T. J.), 1952. - Brain abscess due to Cladosporium tri- 
choïdes. Report of the second case due to this organism. Bull. John Hopkins Hospital, Baltimore, 91, 298.

Kimgman (A. M.) et Mescon (H.), 1950. - The periodic acid Schiff Stain for demonstration of fungi in animal tissue. $J$ Bacter., 60, 415.

Lucasse (C.), Chardome (J.) et Magis (P.), 1954. - Mycose cérébrale par Cladosporium trichoïdes chez un indigène du Congo Belge. Note mycologique sur Cladosporium trichoïdes Emmons par Vanbreuseghem (R.). Ann. Soc. Belge Méd. Trop., 34, 475.

MAC Gill (H. C.) et BruecK (J. W.), 1956. - Brain abscess due to Hormodendrum species. Report of a third case. A.M.A. Arch. Path., 62, 303.

MAnson (M. D. E.), 1958. - Chromo-blastomycotic brain abscess in a South African Bantu. Report of a case. South Afric. J. Lab. a. Clin. Med., 4, 283.

Mariat (F.). - Sur le diagnostic mycologique des principales mycoses du système nerveux. La Semaine des Hôpitaux, Path. et Biol., Arch. de Biol. Méd., $33^{e}$ A, VIII/3, P. et B. $901 /$ B. 281.

Nagahata (M.), 1958, - Chromoblastomycosis of the brain caused by Hormodendrum pedrosoï. Report of a case. Paediatr. Univ. Tokyo, 2, 75.

Netto (A. S. F.), de Britto (T.) et de Almeida (F. P.), 1953. - Cromomicose do sistema nervoso ; estudo anatomico-clinico de um caso. Arq. neuropsiq., 11, 265.

RILeY (O.) et MANN (S.), 1960. - Brain abscess caused by Cladosporium trichoödes. Review of three cases and report of fourth case. Amer. Jl Clin. Path., 33, 525 .

Ségrétain (G.), Mariat (F.) et Drouhet (E.), 1955. - Sur Cladosporium trichoïdes isolé d’une mycose cérébrale. Ann. Inst. Pasteur, 89, 465.

Silva (M.), 1957. - The parasitic phase of the fungi of chromoblastomycosis: development of sclerotic cells in vitro and in vivo. Mycologia, 49, 318.

SILVA (M.), 1958. - The saprophytic phase of the fungi of chromoblastomycosis: effect of nutrients and temperature upon growth and morphology. Trans. N.Y. Acad. Sci., s. II, 21, 46.

TréJos (A.), 1954. - Cladosporium carrionii n. sp. and the problem of Cladosporia isolated from Chromoblastomycosis. Rev. Biologia Trop., 2, 75.

WARROT, 1961. - Communication orale (Lille).

Watson (K. C.) et Lines (G. M.), 1957. - Brain abscess due to fungus Hormodendrum. South. Afric. Med. Jl, 31, 1081.

WYBEL (R. E.), 1952. - Mycosis of cervical spinal cord following intrathecal penicillin-therapy. Arch. Pathol., 53, 167 (in Mariat, 1957).

Laboratoire de Parasitologie et Zoologie appliquée (Prof ${ }^{r}$ J.-M. DoвY) et Laboratoire d'Anatomie pathologique (Prof ${ }^{r}$ M.-L. CHEVREL) de la Faculté de Médecine et Pharmacie de Rennes 


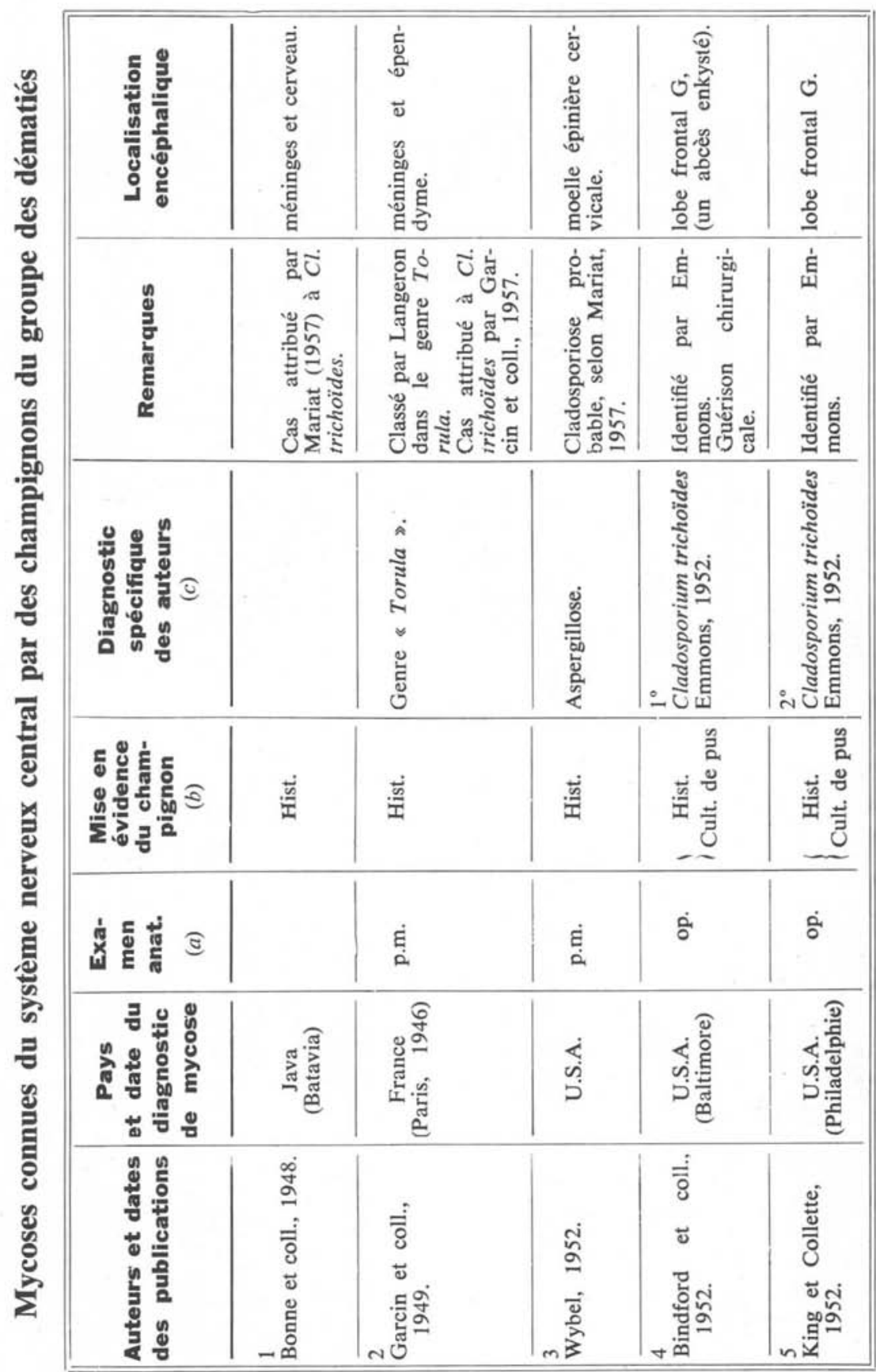

Ann. de Parasitologie, T. XXXVII, $\mathbf{N}^{\circ}$ 4. -1962. 


\begin{tabular}{|c|c|c|c|c|c|}
\hline 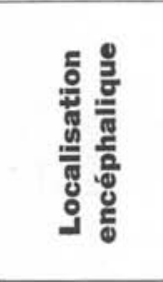 & 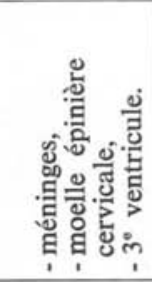 & 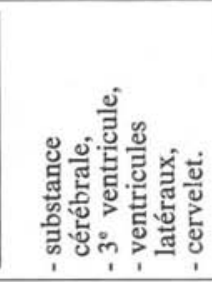 & 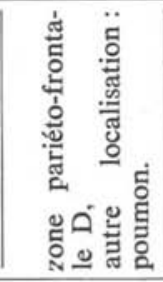 & 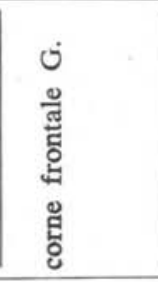 & 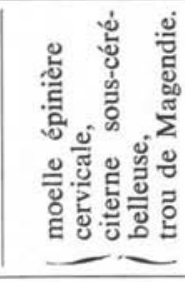 \\
\hline 告 & 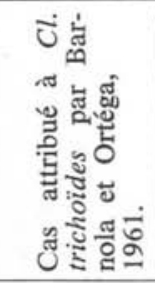 & 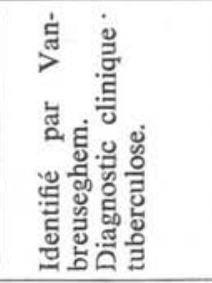 & 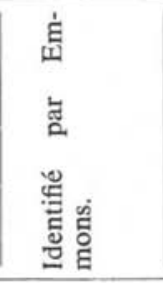 & 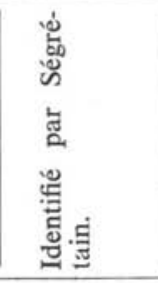 & 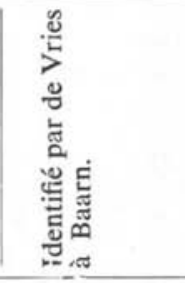 \\
\hline 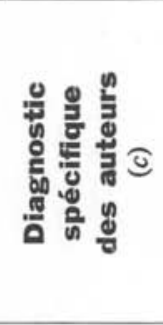 & 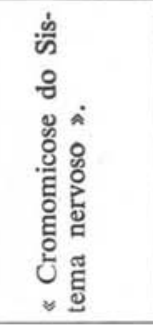 & 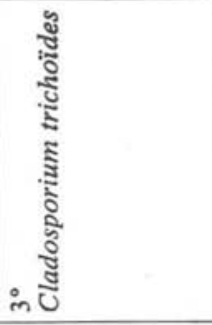 & 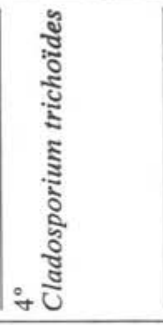 & 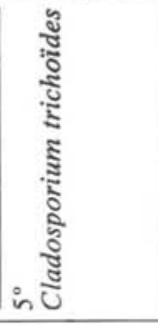 & 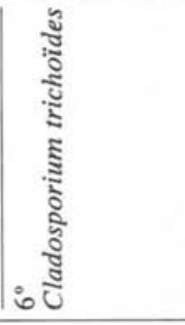 \\
\hline 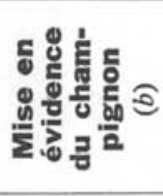 & $\stackrel{\dot{m}}{\frac{a}{x}}$ & 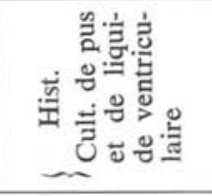 & 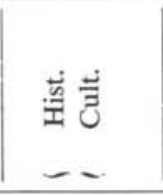 & 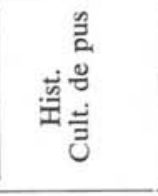 & 离 \\
\hline 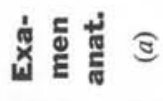 & 豆 & 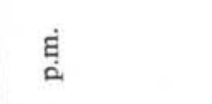 & 㤩 & ல் & ه் \\
\hline 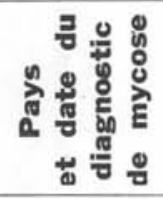 & 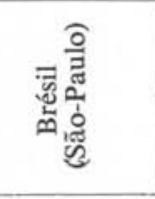 & 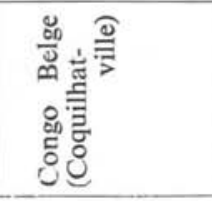 & 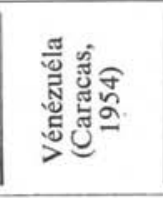 & 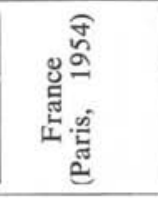 & 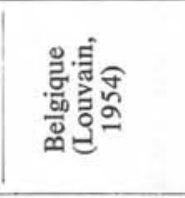 \\
\hline 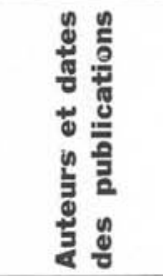 & 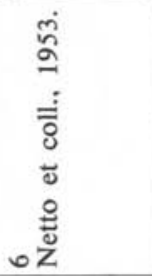 & 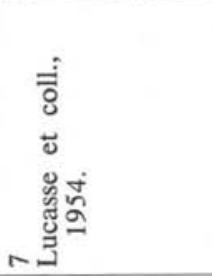 & 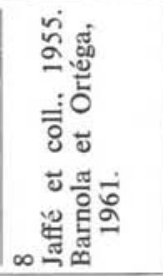 & 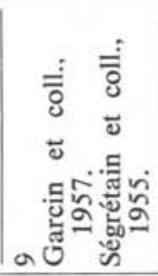 & 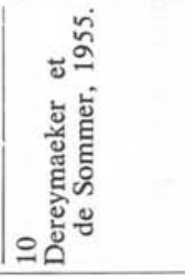 \\
\hline
\end{tabular}




\begin{tabular}{|c|c|c|c|c|}
\hline 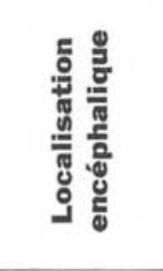 & 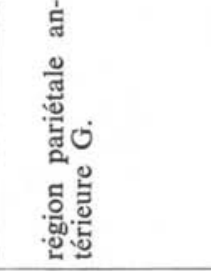 & 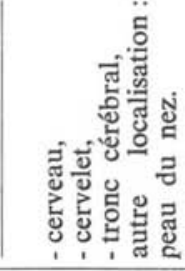 & 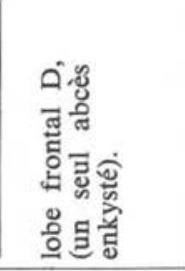 & 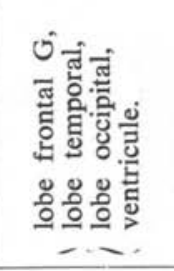 \\
\hline 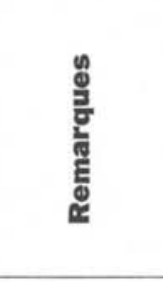 & 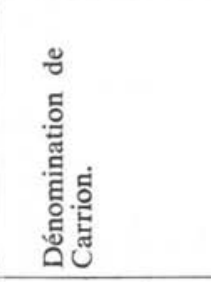 & 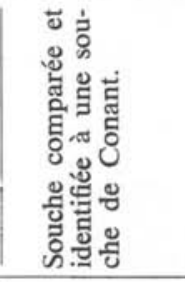 & 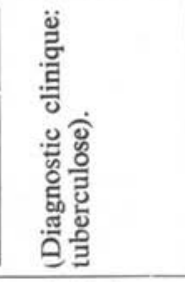 & 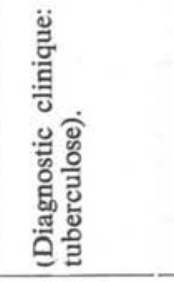 \\
\hline 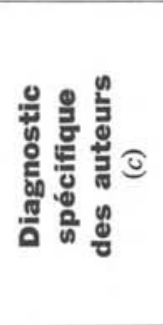 & 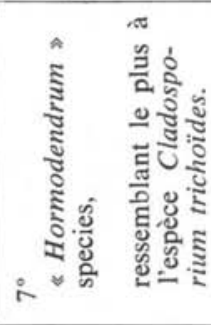 & 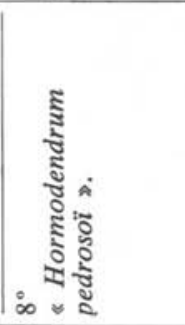 & 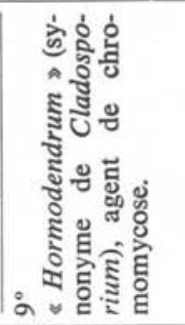 & 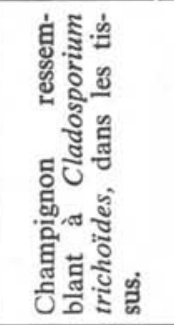 \\
\hline 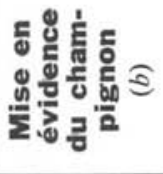 & 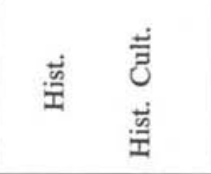 & 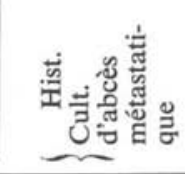 & $\begin{array}{l}\dot{3} \\
\dot{3} \\
\dot{m}\end{array}$ & 蒙 \\
\hline 离 & 豆 & ฉ્મ & ह્ & 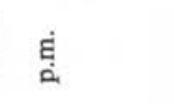 \\
\hline 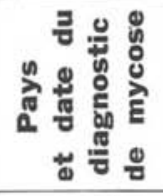 & 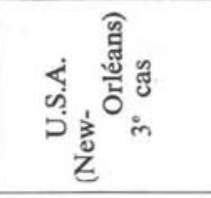 & Б્ & 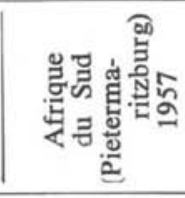 & 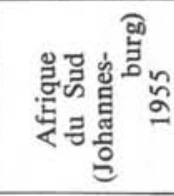 \\
\hline 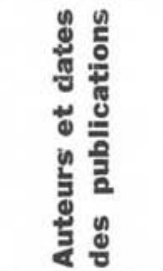 & 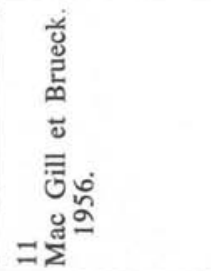 & 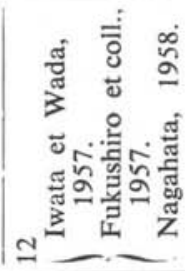 & 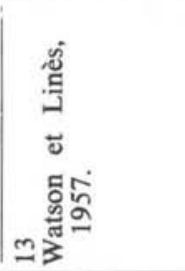 & 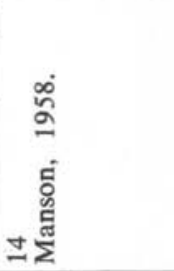 \\
\hline
\end{tabular}


662 M. DOBY-DUBOIS, M.-L. CHEVREL, J.-M. DOBY ET Y. ROBERT

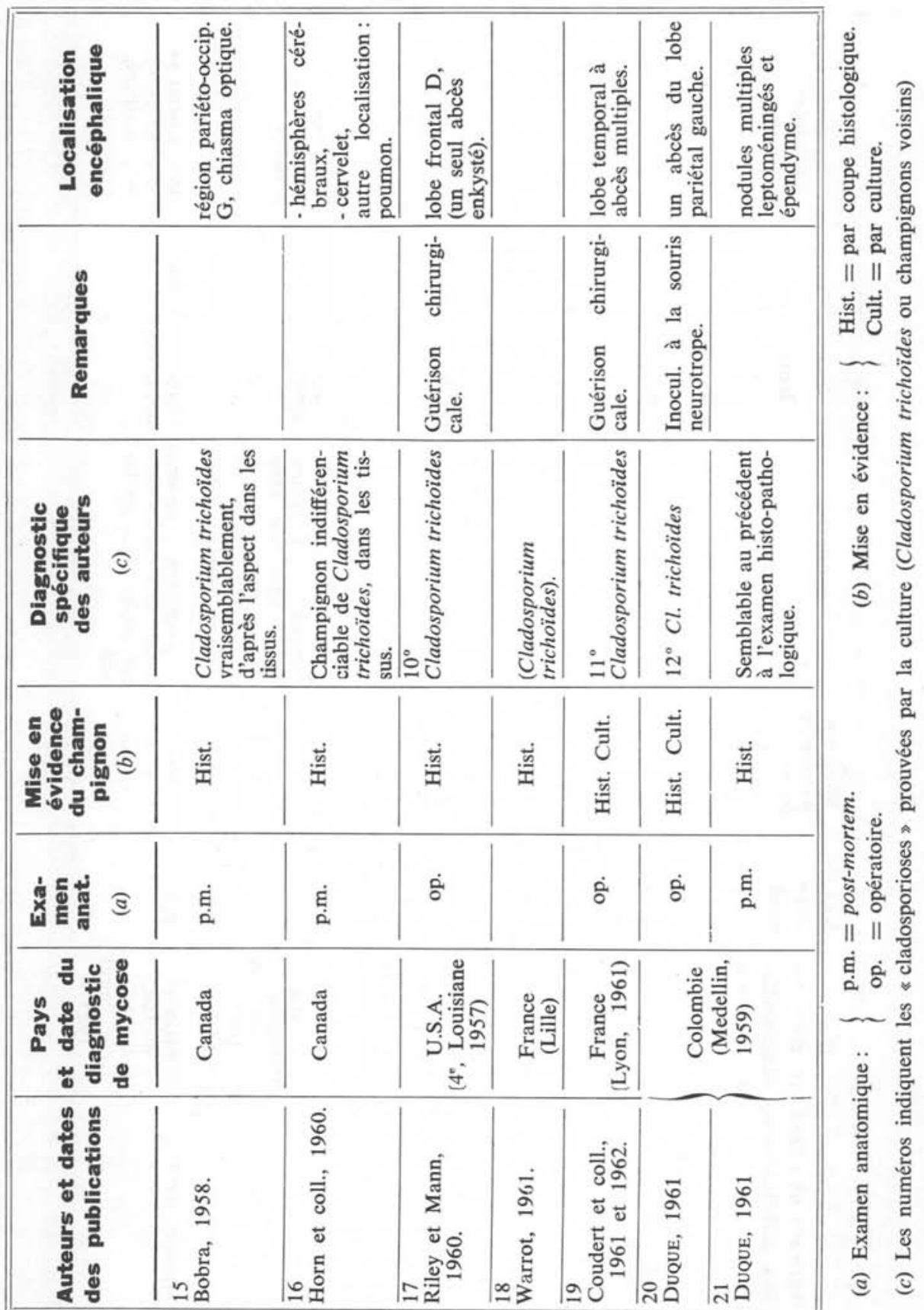

